Trauma Berufskrankh 2009 • 11:5-6 DOI 10.1007/s10039-009-1479-0

Online publiziert: 1. April 2009

๑) Springer Medizin Verlag 2009

\author{
V. Bühren \\ BG-Unfallklinik Murnau, Murnau
}

\title{
Amputation vs. Extremitätenerhalt
}

Die Indikation zum Erhalt schwerstverletzter Extremitäten ist eine der zentralen Kernfragen, seit die Chirurgie systematisch ausgeübt wird. Peter Bamm [1] beschrieb in seinem Werk „Die unsichtbare Flagge“ eindrucksvoll, wie im 2. Weltkrieg unter den Bedingungen des Frontlazaretts die Indikation zur Amputation jeweils angepasst werden musste. Neben den Patientenfaktoren wie Gewebeschaden, Schockzustand und Intervall zur Verletzung spielten hier die äußeren Vorgaben und Transportmöglichkeiten eine wesentliche Rolle. Für offene Fraktursituationen und schwere Weichteilverletzungen war die Opferung der Extremität letztendlich die verbleibende Therapiemöglichkeit, um die Akutsituation zu beherrschen und schwere lebensbedrohliche Infektionen zu vermeiden.

In den folgenden Jahrzehnten - mit Einführung der Antibiotikatherapie, der verbesserten Möglichkeiten der Gefäß- und Nervenrekonstruktion und effektiverer knöcherner Stabilisierungen wurde der Gliedmaßenerhalt zur Nagelprobe einer effizienten traumatologischen Versorgung. Makroreplantationen an der oberen Extremität wurden selbstverständlich, auch für die untere Extremität wurden Kasuistiken mit sinnvollen Ergebnissen berichtet. Die Amputation entwickelte sich mehr und mehr zur Bankrotterklärung des Chirurgen, sei es bei einer nicht mehr machbaren Primärrekonstruktion, vielmehr noch im Verlauf eines nicht erfolgreichen Erhaltungsversuches.

In der Folge führten die mit weiter Indikation durchgeführten Erhaltungsversuche zu einer hohen Rate von verzögerten Amputationen. Lange et al. [4] ver- öffentlichten 1985 eine Serie mit 47 Tibiafrakturen mit schwerstgradiger Weichteil- und Gefäßverletzung, von denen 16 primär und 18 verzögert amputiert wurden, was letztendlich einer Amputationsrate von $72 \%$ entspricht. Bondurant et al. [2] berichteten 1988 über eine Serie von 43 Amputationen nach schweren Unterschenkelverletzungen, von denen 14 primär und 29 verzögert amputiert wurden. Es verstarben 6 Patienten, alle aus der verzögert amputierten Gruppe. Diese Befunde lenkten die Aufmerksamkeit neben der primären Indikation zum Gliedmaßenerhalt wieder auf das zweite wesentliche Entscheidungskriterium, nämlich die Überlebenssicherung des Patienten. Aus den klinischen Studien wurde deutlich, dass die Entscheidung zwischen Überlebenssicherung und Extremitätenerhalt die systemischen Parameter Alter, Verletzungsmuster und Schockzustand zu berücksichtigen hatte, neben den lokalen Parametern mit Gewebeschaden und Ischämie im Zeitablauf.

Um die kritische Entscheidung zwischen Extremitätenerhalt und Amputation bereits primär treffen zu können, wurden Scoresysteme eingeführt, die eine Prognose anhand lokaler und systemischer Parameter erlauben sollten. Am bekanntesten ist der Mangled-Extremity-Severity-Score, der so genannte MESS. Dieser vergab jeweils 1-3 Punkte für die lokale Verletzungsschwere, die Ischämiedauer, den systemischen Schockzustand und das Alter des Patienten. Werte unter 4 sollten mit einer sicheren Erhaltungsmöglichkeit, Werte über 7 mit einer 100\%igen Amputationswahrscheinlichkeit einhergehen. Punktwerte von 4-
6 sollten einen Erhaltungsversuch rechtfertigen. Als Absolutindikationen für eine Amputation wurden Crash-Verletzungen mit mehr als $6 \mathrm{~h}$ dauernder Ischämiezeit und begleitende offensichtliche Komplettausfälle des $\mathrm{N}$. tibialis betrachtet.

2001 wurde aus den USA eine groß angelegte prospektive Studie mit über 600 Patienten mit schweren Verletzungen der unteren Gliedmaße veröffentlicht [3]. Innerhalb der Studie wurden 1/3 der Patienten amputiert, bei $67 \%$ konnte die Gliedmaße erhalten bleiben. Bezogen auf den MESS konnte bei 35\% der Patienten die Gliedmaße erhalten werden, obwohl sie über der Schwelle von 7 Punkten lagen. Umgekehrt musste bei $18 \%$ der Patienten amputiert werden, obwohl sie unterhalb der Schwelle von 4 Punkten lagen. Mehr als die Hälfte der Patienten, die sich primär mit einem kompletten N.-tibialisSchaden präsentierten, entwickelten innerhalb von 2 Jahren wieder eine nutzbare Fußsohlensensibilität. Im Langzeitverlauf ergaben sich für die Wiedererlangung der Arbeitsfähigkeit keine Unterschiede zwischen den rekonstruierten Patienten mit Extremitätenerhalt und den amputierten Patienten.

Der letztgenannte Befund, dass die Amputation im Vergleich zum Erhalt der unteren Extremität nicht zwangsläufig mit einem höheren Verlust an Lebensqualität einhergeht, konnte durch zahlreiche Studien, so auch in Deutschland [5], belegt werden. Nach drittgradig offener Unterschenkelfraktur wurden hierbei keine signifikanten Unterschiede bezüglich Globaleinschätzung der Lebensqualität, der physischen Mobilität und der Möglichkeit zur Selbstversorgung gesehen. Im Ver- 
gleich von primär zu verzögert amputierten Patienten zeigten sich dagegen durchgehend schlechtere Ergebnisse nach fehlgeschlagenen Erhaltungsversuchen.

Heutige Konzepte müssen die vorgenannten Ergebnisse zur langfristigen Lebensqualität einbeziehen. Die frühzeitige Amputation ist eben nicht eine Bankrotterklärung des Chirurgen, sondern kann durchaus mit einer rasch möglichen Rehabilitation die sinnvolle Alternative zu einem lang dauernden Erhaltungsversuch mit konsekutiver sozialer und psychischer Desintegration darstellen. Ein Erhaltungsversuch darf niemals das Leben des Patienten gefährden, es gilt weiterhin „live before limb“.

Einigkeit besteht, dass Scoresysteme zwar eine Entscheidungshilfe sein können, letztendlich aber jeder Einzelfall unter Würdigung einer Vielzahl von Faktoren betrachtet werden muss. Sonderstellungen nehmen v. a. Verletzungen an der oberen Extremität und bei Kindern ein. Der Ischämie-Reperfusions-Schaden ist für diese Konstellationen weniger ausgeprägt, und die funktionelle Rekompensationsmöglichkeit ist deutlich günstiger. Alternative Versorgungsmöglichkeiten ergeben sich über partielle Rekonstruktionen, beispielsweise unter Verwendung nicht mehr originär replantierbarer Amputate. In diesem Heft soll ein Überblick $\mathrm{zu}$ den heute bestehenden Optionen und Alternativen der rekonstruktiven Chirurgie gegeben werden.

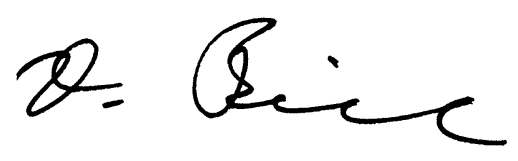

Prof. Dr. V. Bühren

\section{Korrespondenzadresse}

\section{Prof. Dr. V. Bühren}

BG-Unfallklinik Murnau,

Prof.-Küntscher-Straße 8, 82418 Murnau

buehren@bgu-murnau.de

\section{Literatur}

1. Bamm P (1952) Die unsichtbare Flagge. Kösel, München

2. Bondurant FJ, Cotler HB, Buckle R et al (1988) The medical and economic impact of severely injured lower extremities. J Trauma 28(8):1270-1273
3. Bosse MJ, MacKenzie EJ, Kellam JF et al (2001) A prospective evaluation of the clinical utility of the lower-extremity injury-severity scores. J Bone Joint Surg Am 83-A(1):3-14

4. Lange RH, Bach AW, Hansen ST et al (1985) Open tibial fractures with associated vascular injuries: prognosis for limb salvage. J Trauma 25(3):203208

5. Seekamp A, Regel G, Ruffert S et al. (1998) Amputation oder Rekonstruktion bei der III B und III C offenen Unterschenkelfraktur. Unfallchirurg 101(5):360-369 